\title{
The role of 4D flow MRI for clinical applications in cardiovascular disease: current status and future perspectives
}

\author{
Baiyan Zhuang ${ }^{1}$, Arlene Sirajuddin ${ }^{2}$, Shihua Zhao ${ }^{1}$, Minjie Lu ${ }^{1,3}$ \\ ${ }^{1}$ Department of Magnetic Resonance Imaging, Fuwai Hospital, State Key Laboratory of Cardiovascular Disease, National Center for Cardiovascular \\ Diseases, Chinese Academy of Medical Sciences and Peking Union Medical College, Beijing, China; ${ }^{2}$ National Heart, Lung and Blood Institute \\ (NHLBI), National Institutes of Health (NIH), Bethesda, MD, USA; ${ }^{3}$ Key Laboratory of Cardiovascular Imaging (Cultivation), Chinese Academy of \\ Medical Sciences, Beijing, China
}

Contributions: (I) Conception and design: M Lu; (II) Administrative support: S Zhao; (III) Provision of study materials or patients: B Zhuang; (IV) Collection and assembly of data: B Zhuang; (V) Data analysis and interpretation: B Zhuang, A Sirajuddin; (VI) Manuscript writing: All authors; (VII) Final approval of manuscript: All authors.

Correspondence to: Minjie Lu, MD, PhD. Department of Magnetic Resonance Imaging, State Key Laboratory of Cardiovascular Disease, Fuwai Hospital, National Center for Cardiovascular Diseases, Chinese Academy of Medical Sciences and Peking Union Medical College, Beijing 100037, China; Key Laboratory of Cardiovascular Imaging (Cultivation), Chinese Academy of Medical Sciences, Beijing, China. Email: coolkan@163.com.

\begin{abstract}
Magnetic resonance imaging (MRI) four-dimensional (4D) flow is a type of phase-contrast (PC) MRI that uses blood flow encoded in 3 directions, which is resolved relative to 3 spatial and temporal dimensions of cardiac circulation. It can be used to simultaneously quantify and visualize hemodynamics or morphology disorders. 4D flow MRI is more comprehensive and accurate than two-dimensional (2D) PC MRI and echocardiography. 4D flow MRI provides numerous hemodynamic parameters that are not limited to the basic 2D parameters, including wall shear stress (WSS), pulse wave velocity (PWV), kinetic energy, turbulent kinetic energy (TKE), pressure gradient, and flow component analysis. 4D flow MRI is widely used to image many parts of the body, such as the neck, brain, and liver, and has a wide application spectrum to cardiac diseases and large vessels. This present review aims to summarize the hemodynamic parameters of 4D flow MRI technology and generalize their usefulness in clinical practice in relation to the cardiovascular system. In addition, we note the improvements that have been made to 4D flow MRI with the application of new technologies. The application of new technologies can improve the speed of 4D flow, which would benefit clinical applications.
\end{abstract}

Keywords: Magnetic resonance imaging (MRI); cardiovascular system; hemodynamics

Submitted Nov 04, 2020. Accepted for publication Apr 23, 2021.

doi: 10.21037 /qims-20-1234

View this article at: http://dx.doi.org/10.21037/qims-20-1234

\section{Introduction}

Transthoracic echocardiography (TTE) is used to assess local blood flow velocity, and is widely used in routine examinations of cardiovascular diseases. This technique has many advantages, including its wide availability and facile operation; however, due to variable probe orientation and variabilities between observers, its reproducibility is limited.
Additionally, TTE can only detect the velocity component, which is parallel to the sensor. Computed tomography (CT) provides a relatively fast three-dimensional (3D) scan with good spatial resolution, which shows the contrast medium's distribution pattern in blood vessels at any given moment. However, currently, CT has limited value in measuring the blood flow velocity (1). The creative time-resolved four-dimensional (4D) phase-contrast (PC) 
Table 1 The difference between 2D PC MRI and 4D flow MRI

\begin{tabular}{lll}
\hline Variable & 2D PC MRI & 4D flow MRI \\
\hline Introduced (year) & 1980s & 1990s \\
Velocity encoding & 1-directional & 3-directional \\
Analysis level & $\begin{array}{l}\text { The direction oriented perpendicular } \\
\text { to the imaging slice }\end{array}$ & Any level of a given vessel \\
Time-resolved & No & Yes \\
Scan time & Short & Long \\
Temporal resolution & Shorter & Around 40 ms \\
Spatial resolution & Lower & Isotropic high spatial resolution \\
Volume coverage & Small & Large \\
Hemodynamic parameters & Low velocity, flow direction (forward flow, & In addition to the basic flow parameters,4D flow MRI also \\
& reverse flow, regurgitation fraction), flow & detects wall shear stress, turbulent kinetic energy, vorticity, \\
& volume & pressure gradient, pulse wave velocity and flow component
\end{tabular}

$\mathrm{MRI}$, magnetic resonance imaging.

magnetic resonance imaging (MRI) technique (referred to hereafter as 4D flow MRI) can overcome the shortcomings of the above-mentioned methods. 4D flow MRI shows $3 \mathrm{D}$ blood flow patterns and hemodynamics with velocity encoding (VENC) along 3 spatial directions. It contributes to understandings of blood flow characteristics in normal and pathologically altered situations, and provides excellent $3 \mathrm{D}$ coverage of both the anatomy and velocity, which produces accurate estimates of the lengths of vessels and hemodynamic information. With the exception of absolute blood flow quantification, 4D flow MRI can estimate helical blood flow, and thus provides a more detailed assessment of cardiovascular diseases (2). Understanding normal and abnormal blood flow patterns of the cardiovascular system will also provide insights into the complex pathophysiological reasons for diseases of the heart and large vessels (3). The present review sought to illustrate the hemodynamics and morphology of blood flow by 4D flow MRI with a focus on the cardiovascular system.

\section{From 2D to 4D}

2D velocity-encoded PC flow MRI (2D flow MRI) was introduced in the late 1980s and gradually developed into a reliable noninvasive blood flow and velocity measurement tool, which has been used to assess stroke (4). It has been widely verified in normal and multiple cardiovascular diseases $(5,6)$. Previous studies have shown that 2D flow
MRI is reliable and comparable to TTE in examining aortic and pulmonary arteries (7). However, the expertise of a skilled operator is required to identify the vessel of interest, and it can only quantify flow velocities perpendicular to the direction of the imaging slice (8). In addition, it is impossible to visualize the precise position before planning the acquisition plane, which limits the veracity of the measurements. For these reasons, 2D flow MRI significantly underestimates flow rate and velocity (9).

Recently, 4D flow MRI was introduced as a new method for visualizing and quantifying multiple flow patterns of the heart and vessels (10). 2D flow MRI has 1 orientation, while 4D flow MRI allows VENC in 3 principal spatial directions, and thus appears to be a credible tool that reflects the true pathophysiological state of blood flow. Volume acquisition and measurement of reflux are easy in 4D flow MRI, as it covers the whole heart and does not require specialized cardiac anatomy knowledge or specific imaging planes for acquisition (11). Another advantage of 4D flow MRI is that the analysis plane can be set anywhere within the acquisition area offline (8). One study of healthy volunteers reported that the flow parameters quantified by 4D flow MRI can be accurately reproduced (12). 4D flow MRI is thus a suitable technology for comprehensively evaluating the blood flow and energy distribution of the heart and great vessels in healthy people and in patients with cardiovascular disorders (3). Table 1 compares 2D PC MRI measurements and $4 \mathrm{D}$ flow data. 


\section{Features of 4D flow MRI}

4D flow MRI data are generally acquired during free breathing, and retrospective electrocardiogram vector control with an end-expiratory navigation gate is applied to the cross 3-way blood flow coding gradient-echo pulse sequence (13). 4D flow MRI can retrospectively evaluate flow information for the entire vascular system from any image plane, as both the anatomy and the flow information are applied to every pixel in a $3 \mathrm{D}$ volume. 4D flow MRI further has the ability of time-resolved cine velocity acquisition. It should be noted that this time dimension does not reflect real time, but is an effective average collected from many heart cycles. Thus, any unstable or pulsating changes in blood flow are not overly influential. Multiple techniques are used in 4D flow MRI data visualization, including streamline, isosurface, vector field, and volume rendering. Additionally, volumetric acquisition helps to better quantify complex cardiac blood flow (14). In addition to providing traditional 2D PC indexes, including flow direction, forward flow volume, reserve flow volume, regurgitation fraction, and peak velocity, 4D flow MRI also comprehensively provides a retrospective functional assessment of blood flow and helps visualize various forms of blood flow pathways (1). These multifarious hemodynamic biomarkers, such as turbulent kinetic energy (TKE) (15), wall shear stress (WSS) (16), pulse wave velocity (PWV) (17), vorticity (18), and pressure gradient (19), have potential clinical application value. The definitions and applications of these biomarkers are displayed in Table 2 and Figure 1.

\section{Application to great vessels and cardiac diseases}

To date, 4D flow MRI has been used in intracranial and cardiac vascular imaging. In relation to the cardiovascular system, 4D flow MRI is mainly used for congenital heart disease (CHD), but it also has important applications in other cardiovascular diseases, such as aortic aneurysm, aortic stenosis, pulmonary hypertension, and cardiac valvular disease (20) (see Table 3).

\section{Great vessel diseases}

\section{Aortic disease (stiffness, aneurysm, coarctation, dissection, and Marfan syndrome)}

Aortic stiffness is an early sign of functional change, arterial hemodynamic remodeling, and cardiovascular aging. PWV, as detected by 4D flow MRI, has recently been used for full volume assessments of the aorta. It has been shown to be highly accurate in both healthy subjects and patients with complicated aortic geometries (21). PWV is defined as the propagation distance of the pressure wave between 2 anatomical locations divided by the propagation time. Harloff $e t a l$. used the aortic PWV detected by 4D flow MRI to measure aortic stiffness (22) and found that as age increased, $\mathrm{PWV}$ increased from $4.93 \pm 0.54$ to $8.06 \pm 1.03 \mathrm{~m} / \mathrm{s}$. Additionally, PWV was significantly higher in males than females $(\mathrm{P}<0.001)$. Atherosclerosis or size changes of the arteries can cause complex geometry curvature or heterogeneous stiffness along the arterial tree. Compared to 2D PC-based methods, 4D flow PWV is more suitable for complex diseases.

Aortic aneurysms often involve the ascending aorta (AAo). When the diameter of the AAo surpasses $6 \mathrm{~cm}$, peeling or rupture may occur, increasing the life-time risk of aneurysms (23). However, it is almost impossible to predict the progression of an aneurysm, and peeling and rupture can occur when the diameter is less than $6 \mathrm{~cm}$. Measurements of aortic hemodynamics and changes of WSS and their relationship to aortic dimensions may provide further insights into the development of aneurysms and assist in assessing the risk of dissection (24). Bürk et al. (25) examined the correlation between the AAo and aortic arch's 3D flow patterns and vascular wall parameters in patients with concomitant AAo dilatation, and found that the occurrence and intensity of the AAO helix and vortex flow were significantly higher in dilated AAo patients compared to normal controls, while the WSS at the peak systolic of the AAo and aortic arch was decreased $(\mathrm{P}=0.0157-0.0488)$. The AAo diameter was positively correlated with peak velocities $(\mathrm{P}<0.04)$, and was negatively correlated to WSS $(\mathrm{P}<0.03)$ (see Figure 2).

4D flow MRI of the aorta can also be applied to aortic coarctation (CoA). 4D flow MRI can assess changes in the hemodynamics and flow-derived vessel wall parameters of CoA patients (27). In CoA patients, noninvasive 4D flow MRI may provide an alternative to invasive catheterization (28). Saitta et al. (29) developed a novel 4D flow MRI algorithm on the basis of a finite element solution of the Poisson pressure equation to acquire relative distributions of pressure by $4 \mathrm{D}$ flow data, so that it can be widely used in clinical practice.

In an ex vivo porcine experiment, 4D flow MRI was shown to be useful in aortic dissection and to accurately assess blood flow in the real and false lumen. This could assist in determining the area of the first or second tear, or 
Table 2 Summary of hemodynamic parameters and their implication

\begin{tabular}{|c|c|c|c|}
\hline Parameter & Definition & Physiological implication & Note \\
\hline PWV (m/s) & $\begin{array}{l}\text { The speed at which a pulse wave } \\
\text { traveled along a vessel; usually } \\
\text { several times quicker than the blood } \\
\text { flow velocities }\end{array}$ & $\begin{array}{l}\text { A marker of vascular stiffness; mirrors the } \\
\text { overall compliance of vessels; assists in } \\
\text { the interpretation of specific sites of focal } \\
\text { diseases, such as atherosclerotic plaque } \\
\text { and aneurysms }\end{array}$ & $\begin{array}{l}\text { Boundary layer analysis of small-scale } \\
\text { vascular velocity profile; correlated to } \\
\text { temporal resolution }\end{array}$ \\
\hline WSS (Pa) & $\begin{array}{l}\text { Friction and shear stress imposed by } \\
\text { tangential liquid and viscosity along } \\
\text { blood vessel wall }\end{array}$ & $\begin{array}{l}\text { Demonstrated endothelial function; } \\
\text { correlated to vascular disorders, such as } \\
\text { arteriosclerosis and the stability of plaque }\end{array}$ & $\begin{array}{l}\text { Depends on the contour of the wall } \\
\text { boundary; may be an underrated } \\
\text { attribute for several reasons: finite } \\
\text { partial -volume effect, low space } \\
\text { resolution, digital derivation of the } \\
\text { flow distribution }\end{array}$ \\
\hline TKE (mJ) & $\begin{array}{l}\text { A measure of energy loss in disturbed } \\
\text { flow; represents the energy } \\
\text { transformed into heat because of } \\
\text { turbulence flow }\end{array}$ & $\begin{array}{l}\text { Blood flow becomes turbulent in local } \\
\text { narrows because of aortic valves stenosis } \\
\text { or aortic coarctation; higher TKE with } \\
\text { similar flow velocities indicates a more } \\
\text { serious stenosis situation }\end{array}$ & $\begin{array}{l}\text { The sensitivity is affected by VENC; } \\
\text { mitigated by a multipoint approach } \\
\text { with } 3 \text { different VENCs per direction }\end{array}$ \\
\hline Vorticity $\left(s^{-1}\right)$ & $\begin{array}{l}\text { Local rotation of blood particles; } \\
\text { derivative quantity of the velocity field; } \\
\text { a measurement of velocity gradients } \\
\text { in different spaces can describe the } \\
\text { vortex flow }\end{array}$ & $\begin{array}{l}\text { Related to cardiac function, especially } \\
\text { diastolic dysfunction; evaluates } \\
\text { complicated congenital intracardiac } \\
\text { malformations; high vorticity values cor- } \\
\text { related with a high risk of atherosclerosis }\end{array}$ & $\begin{array}{l}\text { The precise position and appearance } \\
\text { of the vortices in the cardiac cycle } \\
\text { should be considered; depends on } \\
\text { chamber geometry; increased vorticity } \\
\text { could result in viscous energy loss }\end{array}$ \\
\hline $\begin{array}{l}\text { Components } \\
\text { of dynamic } \\
\text { ventricular } \\
\text { flow }(\%)\end{array}$ & $\begin{array}{l}\text { Including direct flow, retained inflow, } \\
\text { delayed ejection flow, and residual } \\
\text { volume }\end{array}$ & $\begin{array}{l}\text { Can detect LV reconstruction and } \\
\text { dysfunction }\end{array}$ & $\begin{array}{l}\text { Sensitive to noise and systematic } \\
\text { errors }\end{array}$ \\
\hline
\end{tabular}

PWV, pulse wave velocity; WSS, wall shear stress; TKE, turbulent kinetic energy; KE, kinetic energy; VENC, velocity encoding; LV, left ventricular; RV, right ventricular.

detect thrombosis in the false cavity (30). Allen et al. found that 4D flow MRI can be applied to identify the upper peel flap window. 4D flow MRI can also be applied as an auxiliary method of B-type peeling MRI or magnetic resonance angiogram to assess the flap window. Additionally, the basic 4D flow MRI hemodynamic parameters, including stroke volume (SV), flow velocity, and the helical flow in the false cavity, are all associated with the expansion rate of the aorta. When implementing endovascular repairs, the ability to determine hemodynamic active fenestrations may allow 4D flow MRI to complement the CT angiography anatomical distinction (31).

Geiger et al. (32) studied general and segmental hemodynamic aortic changes in Marfan syndrome (MFS) 


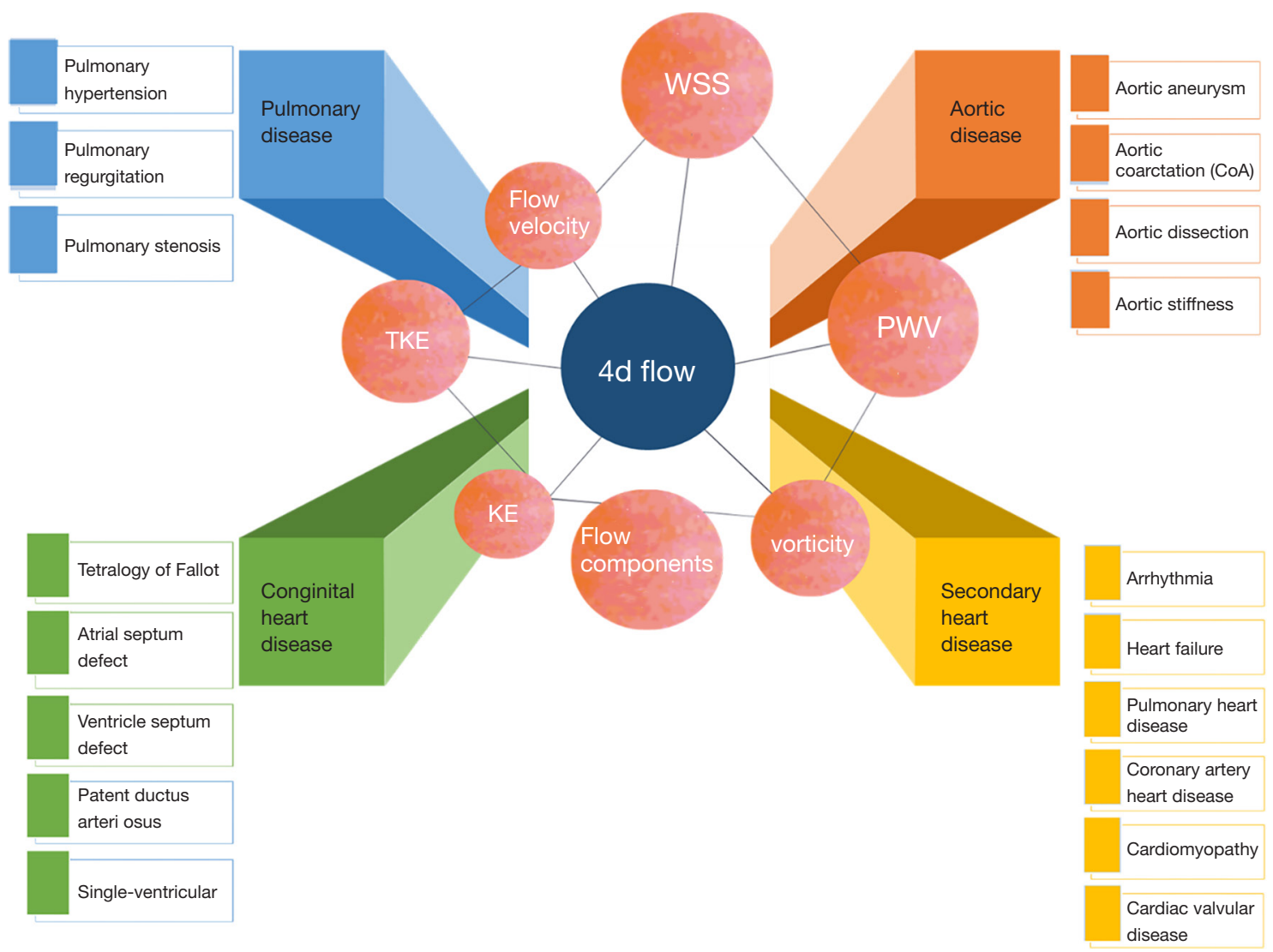

Figure 1 Hemodynamic parameters and clinical applications of 4D flow MRI. MRI, magnetic resonance imaging; WSS, wall shear stress; KE, kinetic energy; TKE, turbulent kinetic energy; PWV, pulse wave velocity.

patients and found that while the aortic hemodynamics remained stable overall, mild changes in the local descending aorta (DAo) appeared at an early age and became much more evident over time (see Figure 3). Segmental WSS was found to be lower in the inner proximal DAo segment of MFS patients (see Figure 4), which is related to increased abnormal vortex/helix flow patterns.

\section{Hemodynamic differences}

4D flow MRI can be applied to evaluate the flow of the vena cava and right atrium (RA) inflow tract. Wehrum et al. (26) provided reference values for the quantification of vena cava blood flow based on 4D flow MRI, and found that age had a significant effect on cava blood flow and RA hemodynamics. A clockwise rotating helix was observed in young patients while turbulence was significantly more frequent in elderly patients (see Figure 5). In the future, when using 4D flow MRI to evaluate the pathological state of the heart, the effect of aging should be considered.

\section{Pulmonary bypertension}

It is viable to measure pressure in the artery by $4 \mathrm{D}$ flow MRI. Pulmonary hypertension is diagnosed by measuring the mean pulmonary artery pressure (mPAP) through a right heart catheter. However, a 4D flow MRI analysis can also accurately estimate mPAP from the duration of the blood flow vortex in the pulmonary artery (PA) (33). In previous studies, the duration of the PA vortex (expressed as a percentage of the cardiac cycle) has been shown to be well correlated with the invasively measured mPAP (34). Kheyfets et al. (35) developed a multivariable model that included PA relative flow characteristics to detect pulmonary vascular resistance by $4 \mathrm{D}$ flow MRI. They noted that combining the vorticity of the main PA (MPA) and the right $\mathrm{PA}(\mathrm{RPA})$ in the multivariate model enabled it to predict $85 \%$ of mPAP abnormities. 
Table 3 Summary of selected studies applying 4D flow MRI to the heart and vessels

\begin{tabular}{|c|c|c|c|}
\hline Anatomy & Diseases & Indices & Findings \\
\hline \multirow{6}{*}{ Heart } & Atrial fibrillation & $\begin{array}{l}\text { Peak velocity; } \\
\text { time to-peak velocity }\end{array}$ & Peak velocity is more sensitive to the changes of flow \\
\hline & Pulmonary heart disease & LV vorticity & Damaged LV vortex formation, impaired LV vorticity \\
\hline & Repaired TOF & Flow pattern; KE; vorticity & Altered vortical patterns; increased KE \\
\hline & Atrial septum defect & $\begin{array}{l}\text { Blood flow; shunt fraction } \\
\text { and volume }\end{array}$ & $\begin{array}{l}\text { Pulmonary and systemic blood flow associated well with } \\
\text { each other in multiple levels }\end{array}$ \\
\hline & Single ventricle & SPCF & SPCF could be a risk factor of increased morbidity and mortality \\
\hline & Dilated cardiomyopathy & Vorticity & Vorticity enhanced \\
\hline \multirow{6}{*}{$\begin{array}{l}\text { Large } \\
\text { vessels }\end{array}$} & Bicuspid aortic valve & WSS; blood flow & Unsymmetrical elevated WSS in aortic valve; appeared eccentric flow \\
\hline & Aortic aneurysm & $\begin{array}{l}\text { Flow patterns; } \\
\text { peak WSS }\end{array}$ & $\begin{array}{l}\text { Higher helix and vortex and lower peak systolic WWS appeared in } \\
\text { the AAo }\end{array}$ \\
\hline & Aortic coarctation & WSS; vortices & Increased WSS and vortices \\
\hline & Aortic dissection & $\begin{array}{l}\text { Stroke volume; } \\
\text { expansion rate }\end{array}$ & Stroke volume and expansion rates are greater in the true lumen \\
\hline & Pulmonary hypertension & Vortex duration & $\begin{array}{l}\text { Vortex duration correlates well with mean of pulmonary artery } \\
\text { pressure which is tested invasively }\end{array}$ \\
\hline & $\begin{array}{l}\text { Pulmonary regurgitation vs. } \\
\text { stenosis }\end{array}$ & $\mathrm{KE}$ & Higher KE in pulmonary stenosis \\
\hline
\end{tabular}

MRI, magnetic resonance imaging; KE, kinetic energy; PWV, pulse wave velocity; WSS, wall shear stress; EL, energy loss; SPCF, system-to-pulmonary collateral blood flow; TOF, tetralogy of Fallot; LVOT, left ventricular outflow tract; AAo, ascending aorta; CoA, aortic coarctation; LA, left atrial; LV, left ventricular.

\section{$P A$ regurgitation vs. $P A$ stenosis}

4D flow MRI has been shown to be a noninvasive technique that evaluates right ventricular $(\mathrm{RV})$ reconstruction due to load stress caused by PA regurgitation (PR) or PA stenosis (PS). Fernandes et al. conducted a study to quantitatively assess kinetic energy (KE), power output, and power loss parameters on the basis of 4D flow MRI during RV remodeling after PS and PR (36). They found that: (I) the RV KE in systole and diastole changed significantly in PS and PR; (II) compared to patients in the PS group, those in the PR group had a greater capacity load and cardiac output, which led to an increase in KE; and (III) the power output rate of the right ventricle in PR was greater than that in PS. 
A Vortex flow
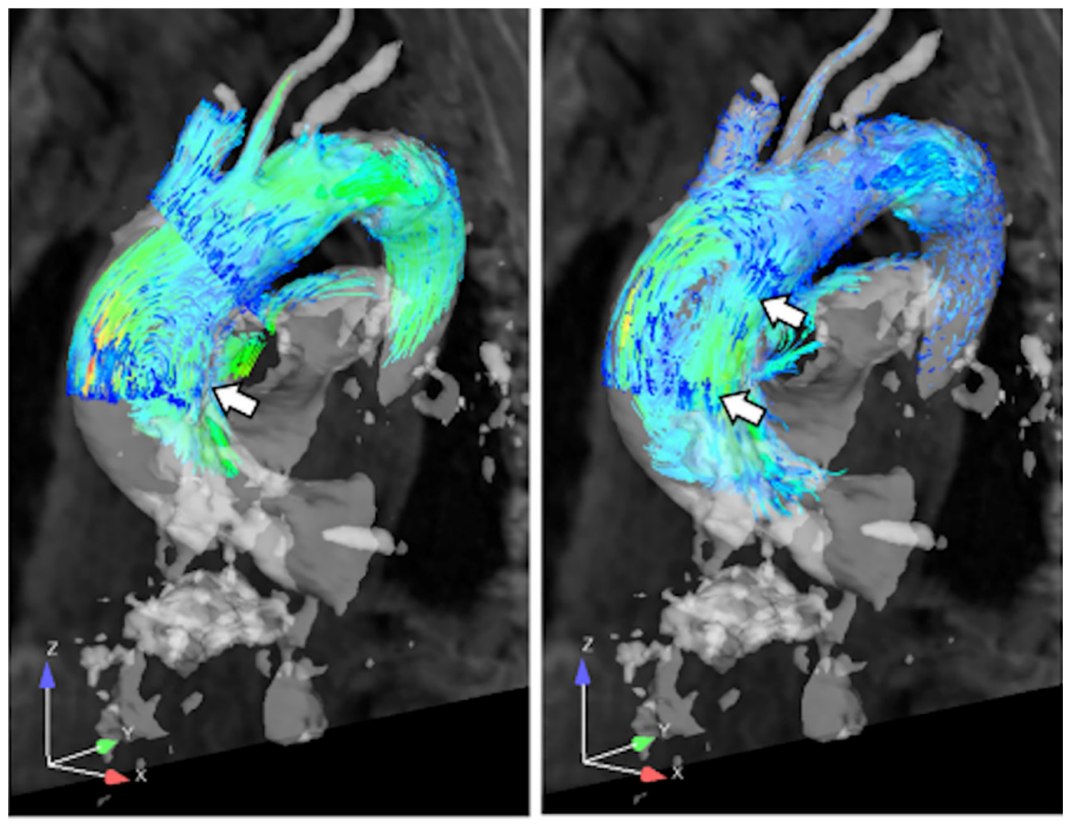

B Helix flow
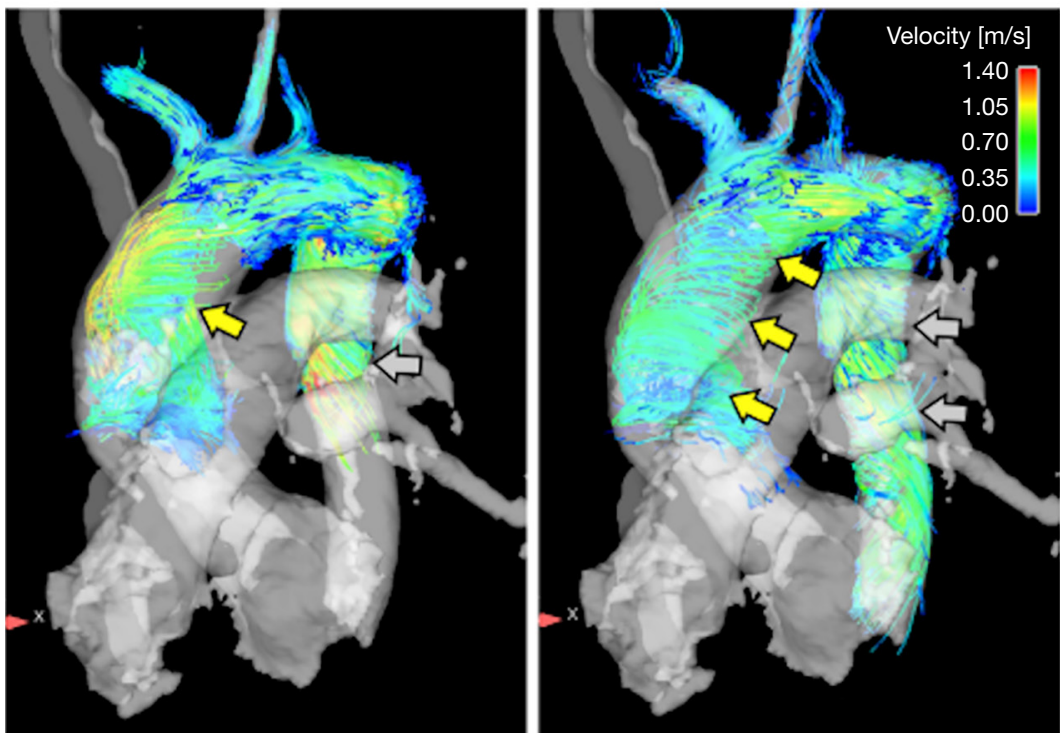

Figure 2 Examples of vortex and helix flow. (A) Early (left photo) and late (right photo) time-resolved particle trajectories in systole show the formation of a large flow vortex (white arrows, grade $=1.5$, diameter $=41 \mathrm{~mm}$ ) in an aneurysm patient. (B) Evolution of helix flow (yellow arrows) in the ascending aorta (grading $=2$, diameter $=48 \mathrm{~mm}$ ) and in the descending aorta (gray arrows) in another patient (26).

\section{Cardiac diseases}

\section{Arrbythmia}

Assessing the atrial blood flow rate of atrial fibrillation (AF) patients is essential, as AF is correlated with an increased risk of embolic stroke (10). Markl et al. (37) undertook a visual and quantitative assessment of left atrial (LA) blood flow, LA stasis, peak velocity, and time to-peak (TTP) velocity using 4D flow MRI, and found that reducing LA peak velocity and increasing the area of LA stagnation were more susceptible to changes in LA flow than was TTP. Cibis et al. (38) used electrical cardioversion to study 4D 

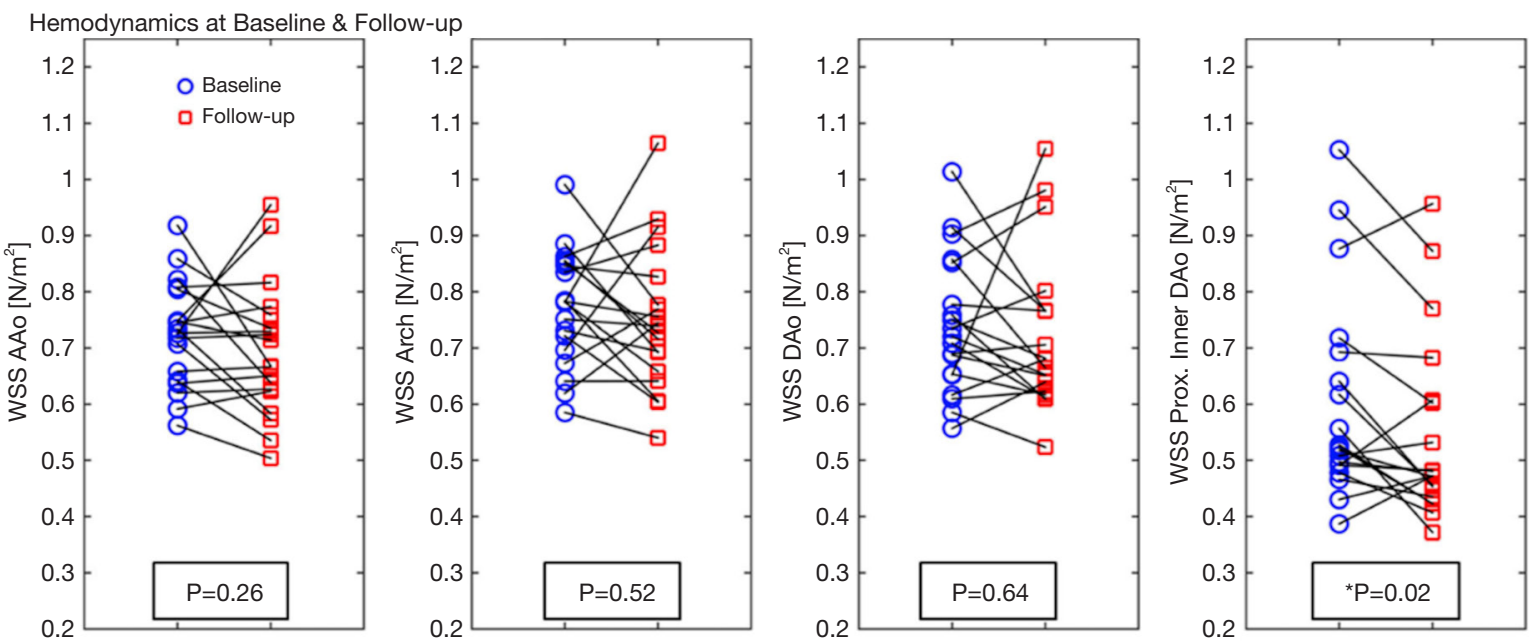

Figure 3 The average systolic WSS of the AAo, arch, DAo, and proximal segment of the DAo at baseline and during follow-up in patients with MFS. *, represents a significant difference. WSS, wall shear stress; AAo, ascending aorta; DAo, descending aorta; MFS, Marfan syndrome (32).
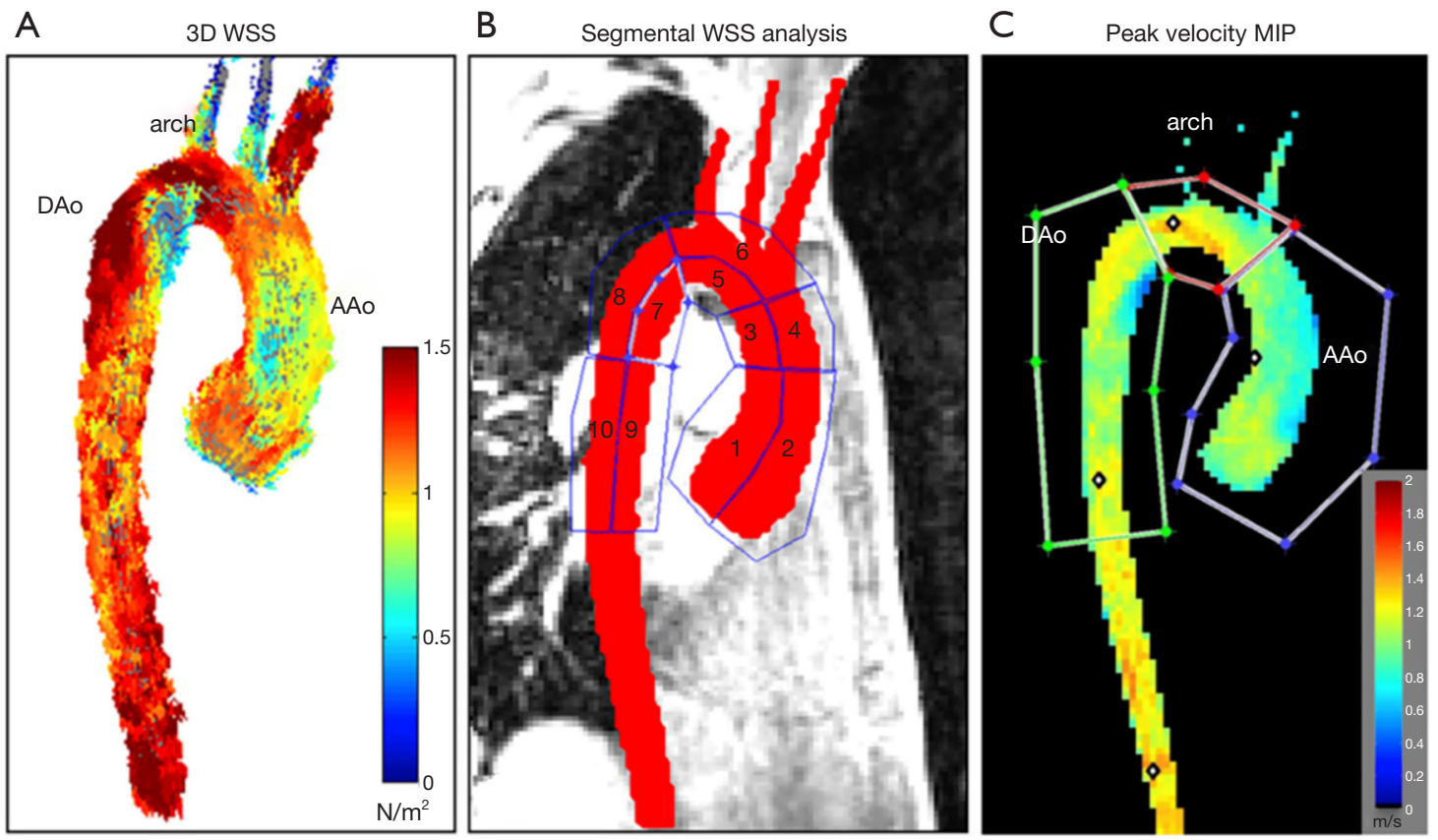

Figure 4 Segmental WSS and peak velocity of aorta. (A) 3D WSS across the entire aortic surface; (B) ten areas applied to segment WSS throughout aorta; (C) peak systolic velocity maximum intensity projection. WSS, wall shear stress; AAo, ascending aorta; DAo, descending aorta; MIP, minimum intensity projection (32). 


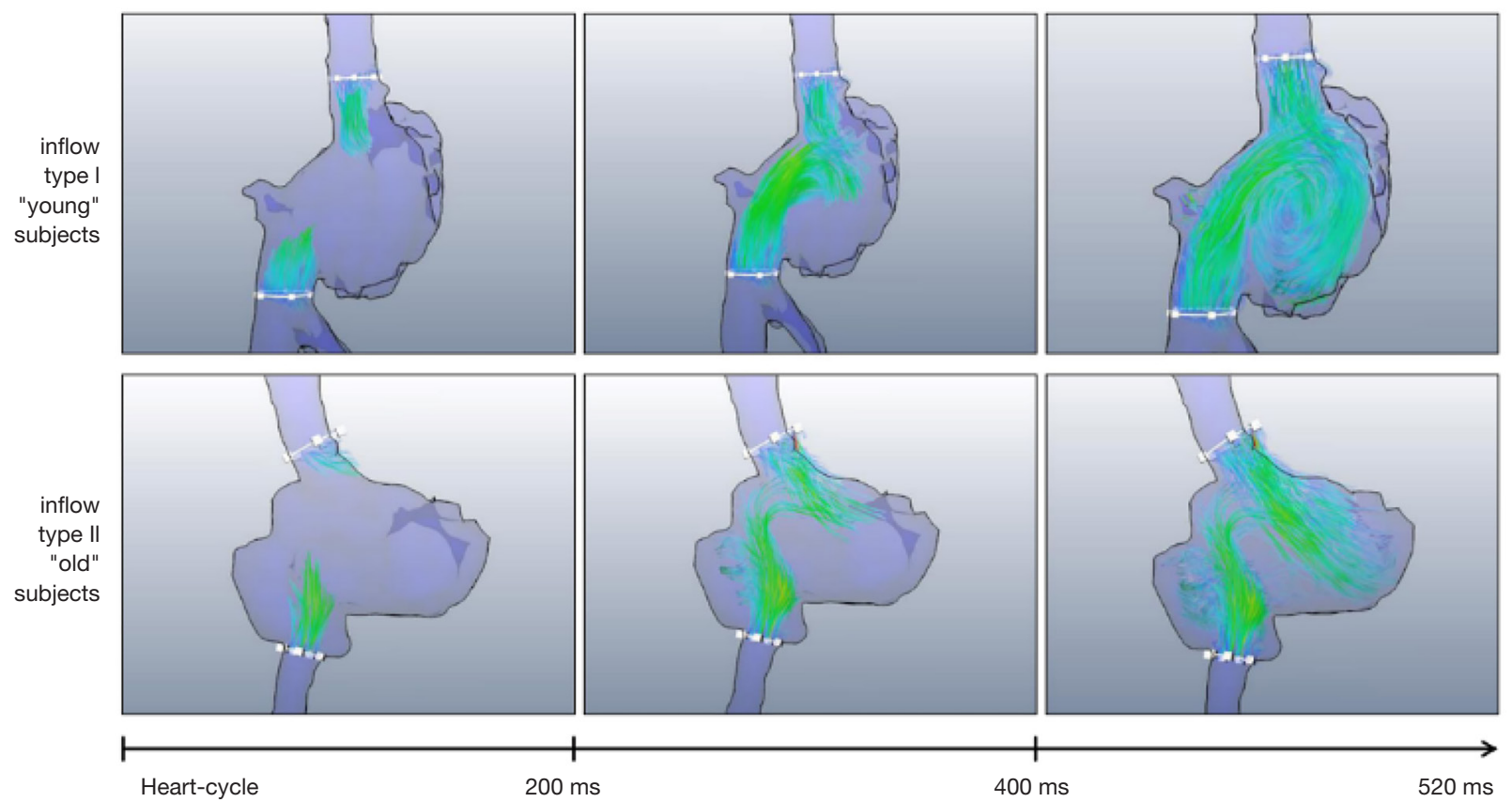

Figure 5 Photos of right atrial filling. A clockwise rotated vortex flow was observed in young patients. In relation to older patients, right atrial filling flow was turbulent; however, even some young patients did not display the formation vortex (25).

LA flow patterns and hemostasis in AF patients. The results showed that the flow form of LA was abnormal after the mechanical function of LA was restored. Further, during LA stunning, there was a significant association between LA blood stasis indicators, and hypercoagulability parameters.

\section{Heart failure}

Left ventricular (LV) dysfunction and remodeling are progressive and can exist early and before obvious clinical symptoms become apparent; thus, an accurate and early screening diagnosis technique is urgently needed. The early detection of the asymptomatic period is the clinical focus of reducing patient morbidity and mortality. To date, KE flow parameters, including vortices and proportions of the functional flow components, have been applied to evaluate the function of atria and ventricles (39). The KE of blood represents the work required to accelerate a certain mass of blood from rest to a specified speed. Even before heart enlarging and malfunction, 4D flow can assess changes in the proportions of flow components and the relevant KE (40). This may enable ventricular dysfunction to be detected in patients in a clinically compensatory stage and provide unique pathophysiological insights into heart failure diagnosis and therapy. Intracardiac KE can provide a novel recognition of cardiac hemodynamic disorder, and can help us to understand and assess heart failure (41). Kanski et al. showed that the average $\mathrm{KE}$ in systole was higher in heart failure patients than in control patients $(2.2 \pm 1.4 v s .1 .6 \pm 0.6 \mathrm{~mJ}$, $\mathrm{P}=0.048)$. The different $\mathrm{KE}$ time curves shown in patients provided a novel conceptual classification of heart failure.

\section{Pulmonary beart disease}

$\mathrm{LV}$ diastolic vorticity is considered to be a sensitive indicator of diastolic dysfunction in pulmonary heart disease. One study (42) investigated LV diastolic vorticity patients with mild to moderate chronic obstructive pulmonary disease and showed that these patients had significant different LV vortex dynamics compared to healthy controls, albeit the LV cardiac function and structure were similar. In addition, as $L V$ vorticity was found to be relevant to RV function, abnormal LV function appears to be caused by LV-RV interdependency effects.

$\mathrm{RV}$ diastolic function can also be assessed using 4D flow MRI, which offers mechanistic insights into RV diastolic dysfunction. Barker's review (43) suggested that 4D flowderived RV blood flow KE assessment could circumvent 
the issues of the current imaging modalities (44). Crandon's study reported that RV energetics provides novel insights into diastolic filling patterns and allows the quantification of $\mathrm{RV}$ diastolic function (45).

\section{Coronary artery beart disease}

Acute myocardial infarction (AMI) leads to a decrease in LV cardiac function and global LV KE resulting from changes of LV hemodynamics. Blood flow KE parameters can offer a new way to diagnose patients with AMI. Corrado et al. (46) speculated that anterior AMI leads to local changes in LV flow. After comparing 4D flow indices between 12 anterior AMI patients and 19 healthy control patients, they found that peak systolic flow velocity was decreased in the midportion and apex $(\mathrm{P}=0.01$ and $\mathrm{P}=0.02$, respectively) of the LV in anterior AMI subjects. Compared to the control patients, the peak diastolic flow velocity of anterior AMI patients was also lower $(\mathrm{P}=0.01)$. Garg et al. (47) found that $\mathrm{LV}$ blood flow $\mathrm{KE}$ was associated with $\mathrm{LV}$ function and that the area of the infarct area was correlated to the rate of $\mathrm{LV} \mathrm{KE}$ in the plane. They also found that AMI patients with $L V$ thrombus have significantly reduced and delayed wash-in of LV (48).

\section{Cardiomyopathy}

Hypertrophic cardiomyopathy (HCM) is related to a hemodynamic change of the left ventricular outflow tract (LVOT). van Ooij et al. (49) conducted a study to quantify 3D hemodynamic changes, including energy loss (EL) and pressure gradients, in the LVOT using 4D flow MRI. They found that LVOT EL $(3.8 \pm 2.5$ vs. $1.5 \pm 0.7 \mathrm{~mW}, \mathrm{P}<0.005)$ and peak systolic pressure gradients $(21 \pm 16$ vs. $9 \pm 2 \mathrm{mmHg})$ were significantly higher in HCM patients than in control patients.

In a study investigating intraventricular filling flow patterns in patients with nonischemic dilated cardiomyopathy and HCM, enhanced vorticity in nonischemic dilated cardiomyopathy was found to be partially related to reduced $\mathrm{LV}$ stiffness. Conversely, impaired vortex formation was considered to be a potential mechanism for HCM diastolic dysfunction and one of the causes of LV concentric remodeling (50).

Patients with dilated cardiomyopathy develop LV remodeling, but their SVs do not differ from those of normal patients in the early stage. However, a study by Eriksson et al. found that the composition of SV was different but the volume was the same. They also found that the proportion of direct flow (through the LV) in 1 cardiac cycle was smaller. Despite being clinically compensated for, these special blood flow paths and energy alterations could still be detected and could be used as subclinical signs of $\mathrm{LV}$ dysfunction (51).

\section{CHD}

4D flow MRI is also important during the follow-up of patients after tetralogy of fallot repair (rTOF) to evaluate the degree of PR and PS, and to assess RV function. François et al. (52) found rTOF patients have different flow patterns in the right heart compared to normal patients. These changes could be summarized as follows: only vortical flow increased in the RA and RV through the diastole, but both helical and vortical flow increased in the PA. These differences may help explain why symptoms increase during exercise in rTOF patients. Sjöberg et al. (53) discovered that RV diastolic peak KE was significantly increased in rTOF patients, while RV systolic KE did not differ significantly between rTOF and control patients even though PR flow volumes increased considerably. They thus assumed that KE could be used as a potential early sign of ventricular dysfunction to guide intervention therapies. Hu et al. (54) found that increased peak WSS and EL in rTOF patients was associated with pulmonary hemodynamic changes in the MPA and RPA. These might be early markers of evolving hemodynamic inefficiency. They believed that a more comprehensive understanding of PA hemodynamics in rTOF might provide greater insights into PA-RV interactions and how they affect RV function.

In cases of heart disease due to congenital malformations, the presence and severity of tricuspid valve regurgitation is independently related to increased morbidity and allcause mortality. A study found that effective flow across the tricuspid valve quantified by 4D flow MRI had a good correlation with the effective flow of the pulmonary vessel quantified by 2D PC MRI. As the velocity is simultaneously encoded in 3 spatial directions, 4D flow MRI is able to measure tricuspid valve flow and grading of tricuspid regurgitation even for patients with complex RV morphology in clinical practice (55).

4D Flow MRI can also be used to track and visualize blood flow (see Figure 6). Chelu et al. (56) assessed the practicability of using 4D flow MRI to detect the blood flow parameters, shunt volume, and shunt fraction related to atrial septum defect. Blood flow measurements were 


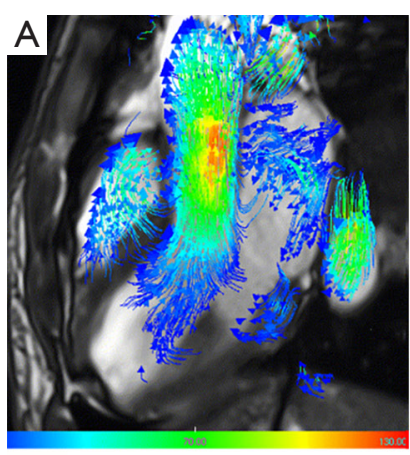

Normal

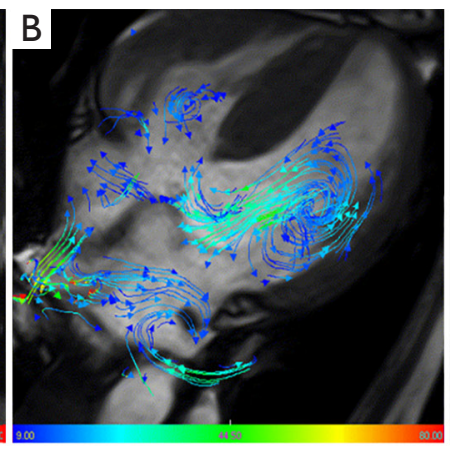

VSD

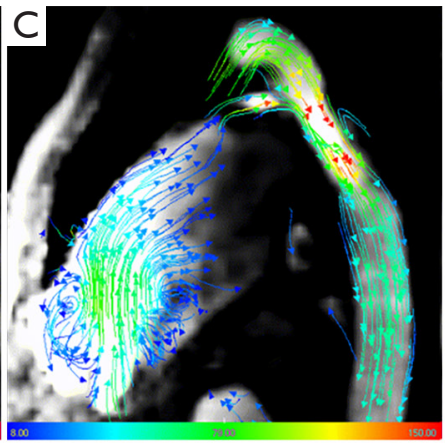

PDA

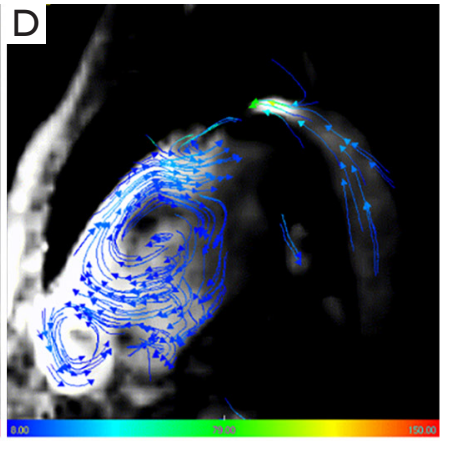

PDA

Figure 6 Representative 4D flow MRI cases of a normal subject and a patient with VSD and PDA from Fuwai Hospital. (A) Streamlines of a normal subject and flow directions are displayed with vector coverage. (B) Streamlines are used to trace blood flow from the left ventricle towards the right ventricle of the VSD. (C,D) Streamlines are used to trace blood flow from the bidirectional shunt between the aorta and the pulmonary artery of the PDA. VSD, ventricular septal defect; PDA, patent ductus arteriosus.

applied at 3 different levels (i.e., the valve, main artery, and peripheral) of pulmonary circulation and systemic circulation. The results showed that measurements of the pulmonary and systemic blood flow at multiple levels correlated well with each other regardless of the valve level $(\mathrm{r}=0.885-0.991)$.

For patients with a single ventricle, system-to-pulmonary collateral blood flow (SPCF) could be a dangerous factor that increases morbidity and mortality. 4D flow MRI differs to 2D PC MRI in that it can assess the complicated blood flow of multiple vessels. Research by Valverde $e t$ al. showed that compared to 2D PC acquisition, SPCF measured by 4D flow MRI was simpler, more accurate, and more effective (57).

Fontan surgery is always the first option for patients with congenital heart defects who are unsuitable for double ventricle repair. An understanding of the characteristics of $\mathrm{KE}$ in a single ventricle could enhance our knowledge of the hemodynamic causes of damaged function in these patients and could help prevent and treat potential complications. Patients with a Fontan cycle were found to have lower peak diastolic KE (58). Further, Kamphuis et al. (59) discovered that the intraventricular EL of Fontan patients was disproportionate to KE. Compared to healthy patients, intraventricular EL was increased in Fontan patients.

\section{Cardiac valvular disease}

For patients with mitral or tricuspid valve regurgitation, the assessment of the severity of the regurgitation is the key to determining whether surgery is necessary. TTE has been widely used; however, it has lower reproducibility for the classification of valve regurgitation. A retrospective study (11) showed that 4D flow MRI was able to quantitatively grade valve regurgitation in such diseases, which could simplify the process of grading and boost the accessibility of cardiac MRI.

4D flow MRI can accurately and repeatedly detect hemodynamic differences by measuring flow, velocity, and direction in patients with congenitally bicuspid or quadricuspid aortic valves (60). The findings of Alvarez's study (61) confirmed that 4D flow MRI correlated well with the conventional standard 2D PC MRI for grading aortic regurgitation (AR). 2D PC MRI is a routine MRI protocol for measuring velocity, net flow, and regurgitation fraction (62); however, it still has limitations, such as a single VENC direction. 4D flow MRI addresses the shortcomings of 2D PC MRI and serves as a new alternative method to MRI for evaluating complex flows through the aortic valve.

Ascending thoracic aorta (AsAo) dilation and the further progression of aortic aneurysms are common, but poorly understood in bicuspid aortic valve (BAV) patients. With its high-resolution 3D acquisition, 4D flow MRI can better characterize the eccentric blood flow of AsAo in BAV patients than can echocardiography. In addition, the 4D flow dataset allows the WSS to be estimated from the near-wall velocity gradient. Rodríguez-Palomares et al. (63) found that different BAV phenotypes have different flow patterns. In patients with the BAV right-left phenotype, the axial WSS in the aortic root was increased, and the circumferential WSS in the middle level and distal level of AAo was also increased in the BAV right-noncoronary 
Table 4 Summary of the limitations of 4D flow

Limitations of $4 \mathrm{D}$ flow
Acquisition
Patients with metal implants are not suitable
The best location to accurately measure flow parameters is
controversial
Postprocessing
Postprocessing and the analysis consume time and labor
The accuracy of blood flow estimation is affected by user
experience
Accuracy
Cannot reliably quantify blood flow when heart cycles are
erratic
Time and space resolution
The spatial resolution and quality are relatively low
Literature support
Long-term follow-up research is lacking

phenotype.

Since the outbreak of the novel 2019 coronavirus (COVID-19), invasive procedures have increased the risk of exposure to viral infections. Thus, it is necessary to assess the severity of valve stenosis through noninvasive procedures. TTE is commonly used in clinical practice, but it may not provide accurate assessments in certain circumstances due to poor acoustic windows and limited windows with narrow fields of view. Transesophageal echocardiography (TOE) is an invasive examination that increases the risk of exposure to COVID-19. Thus, the pressure gradient and the regurgitant jet measured by 4D flow MRI have become particularly useful. Studies have reported that the accuracy of $4 \mathrm{D}$ flow MRI is comparable to that of TOE. In relation to COVID-19, 4D flow is a good alternative for evaluating valve stenosis. 4D flow MRI has become increasingly important in optimizing diagnostic utility with its capabilities of single-sequence, singleacquisition, and retrospective analyses, which allow valve tracking to account for motion throughout the cardiac cycle and the direct measurement of the mitral valve for research and clinical studies (64).

Further, a new 4D flow MRI method with retrospective valve tracking by offline analysis can precisely determine the net flow of all 4 heart valves (i.e., the bicuspid valve, tricuspid valve, pulmonary valve, and aortic valve) in a single scan. This retrospectively triggered scan method can reduce acquisition time, and evaluations of a plurality of valves do not need to be performed separately (65).

\section{Limitations of 4D flow MRI}

4D flow MRI has been shown to be helpful in reflecting the status of different cardiovascular systems; however, it has not been widely used in clinical practice. This is mainly due to its disadvantages (see Table 4). First, the best location to accurately measure flow parameters, such as volume, velocity, and regurgitation, is controversial. Bertelsen et al. (6) found that measuring SV at valve level was the most accurate method; however, Chaturvedi et al. (66) suggested that SV measurement should be obtained at the AAo while AR should be quantified at the sinotubular junction. Future studies should seek to determine the optimal plane for the quantification of the flow parameter with 4D flow MRI. Second, the postprocessing and analysis of 4D flow MRI takes time and labor, which limits its clinical application. The postprocessing time required to measure 4D flow MRI data on the basis of semiautomatic workflow is $40.1 \pm 15.7$ minutes (67). It takes another 30 minutes per patient to manually segment the region of interest in the artery lumen. Third, due to manual intervention in the $3 \mathrm{D}$ segmentation process of the cardiac structure and the correction of the background phase offset, the accuracy of blood flow estimation is further affected by user experience (60). Thus, the analysis workflow needs to be further improved and automated to ensure that the clinical interaction reaches an acceptable level. For example, calculating the center line in the artery lumen and automatically analyzing the plane orthogonal to the center line will reduce the time it takes to manually optimize the plane positioning (68). In addition, 4D flow MRI acquisition usually spans hundreds of heartbeats, making it impossible to study changes in each beat. Thus, MRI cannot quantify blood flow reliably for most diseases with erratic heart cycles. Further, patients with metal implants cannot undergo MRI examinations because of the image artifacts. MRI does not cause any radiation; however, the spatial resolution and quality are relatively low (69). Finally, a systematic review published in 2017 (69) mentioned that of all the included articles on 4D flow MRI, only 4 (9.1\%) were related to diagnostic experiments. Thus, long-term follow-up research needs to be conducted to clarify the clinical usage of hemodynamic parameters derived from 4D flow for risk stratification (3). Future studies should 
seek to determine the accuracy of the existing and novel technologies of 4D flow MRI (10).

\section{The application of new technologies to 4D flow MRI}

As we know, for typical applications, a slow data acquisition coupled with standard navigation-gated 4D flow MRI prolongs scan time and is affected by the patient's respiratory model. Advances in new technologies, including advanced parallel imaging (2), respiratory gating $(70,71)$, radial sampling (71,72), spiral acquisition without acceleration (73), and compressed sensing (74) could reduce the scan time to 5-15 minutes (8). Dyvorne et al. developed a super-speed 4D flow MRI method based on spiral sampling and dynamic compressed sensing. The spiral data were acquired through 3D spiral superposition. Sub-Nyquist sampling was used with a variable-density spiral by an acceleration factor of 6 . A dynamic compressed sensing framework was then applied to rebuilding. Dyvorne et al. demonstrated that the efficient combination of spiral sampling and dynamic compressed sensing rebuilding accelerated the scan time (from about 11 minutes to one breath-hold average). The performance of the hemodynamic parameters, which were examined to compare standard Cartesian 4D flow and 2D PC MRI, were similar.

Additionally, k-space and time dimension generalized auto calibrating partially parallel acquisition (k-t GRAPPA) 4D flow MRI involves spatiotemporal undersampling for the interpolation of missing data in the k-t space, which can reduce the scan time (73-75). Ebel et al. verified that the k-t GRAPPA was not inferior to GRAPPA, and had similar image quality, fewer artifacts, and almost twice the speed (76).

Recently, a new 5-dimensional (5D) flow MRI framework, which exploits data correlated with the cardiac phase and respiratory motion dimensions, has been developed. This framework unites undersampling data acquisition, including multipoint velocity coding, and low-rank image rebuilding, which reduces the scanning time while maintaining image quality (77). The multipoint coding scheme is applied to monitor the average velocity component and the fluctuating velocity component using a noncollinear coding direction, which is similar to the idea of diffusion tensor imaging. Walheim et al., who called this method "5D flow tensor MRI", found that patients with valvular heart disease could undergo a comprehensive blood flow assessment by $5 \mathrm{D}$ flow tensor MRI in 10 minutes (78), and turbulent flow velocity could be plotted in 4 minutes (79).
Moreover, machine learning has been used to segment cardiovascular imaging and reduce the analysis time, which may reduce the time spent on 4D flow measurements (80). The data acquired by deep-learning algorithms has been verified to reflect the data acquired by cardiologists (81-83). Berhane et al. found that deep learning could quickly and automatically implement the $3 \mathrm{D}$ segmentation of the aorta based on 4D flow MRI data, demonstrating its potential to enhance the efficiency of clinical workflows (84).

\section{Conclusions}

As a rising blood flow and velocity quantitative analysis technique, 4D flow MRI can provide 3D blood flow coverage and a retrospective blood flow analysis at any location. 4D flow MRI is important in the detection and guidance of large vessels and cardiac diseases. However, further research needs to be conducted to optimize the clinical application of 4D flow MRI.

\section{Acknowledgments}

Funding: This study was supported in part by research grants from the National Natural Science Foundation of China (No. 81771811 and 81971588), the Construction Research Project of Key Laboratory (Cultivation) of the Chinese Academy of Medical Sciences (No. 2019PT310025), the Clinical and Translational Fund of the Chinese Academy of Medical Sciences (No. 2019XK320063), Capital's Funds for Health Improvement and Research (CFH 2020-2-4034) and the Capital Clinically Characteristic Applied Research Fund (No. Z191100006619021).

\section{Footnote}

Conflicts of Interest: All authors have completed the ICMJE uniform disclosure form (available at http://dx.doi. org/10.21037/qims-20-1234). The authors have no conflicts of interest to declare.

Ethical Statement: The authors are accountable for all aspects of the work in ensuring that questions related to the accuracy or integrity of any part of the work are appropriately investigated and resolved.

Open Access Statement: This is an Open Access article distributed in accordance with the Creative Commons Attribution-NonCommercial-NoDerivs 4.0 International 
License (CC BY-NC-ND 4.0), which permits the noncommercial replication and distribution of the article with the strict proviso that no changes or edits are made and the original work is properly cited (including links to both the formal publication through the relevant DOI and the license). See: https://creativecommons.org/licenses/by-nc-nd/4.0/.

\section{References}

1. Markl M, Kilner PJ, Ebbers T. Comprehensive 4D velocity mapping of the heart and great vessels by cardiovascular magnetic resonance. J Cardiovasc Magn Reson 2011;13:7.

2. van der Geest RJ, Garg P. Advanced Analysis Techniques for Intra-cardiac Flow Evaluation from 4D Flow MRI. Curr Radiol Rep 2016;4:38.

3. Kamphuis VP, Westenberg JJM, van der Palen RLF, Blom NA, de Roos A, van der Geest R, Elbaz MSM, Roest AAW. Unravelling cardiovascular disease using four dimensional flow cardiovascular magnetic resonance. Int J Cardiovasc Imaging 2017;33:1069-81.

4. Chen CY, Li CW, Mak HKF, Lin MF, Chan WP. Combined native magnetic resonance angiography, flow-quantifying, and perfusion-imaging for impending second-stroke assessment. Quant Imaging Med Surg 2019;9:521-9.

5. Wang D, Shao J, Ennis DB, Hu P. Phase-contrast MRI with hybrid one and two-sided flow-encoding and velocity spectrum separation. Magn Reson Med 2017;78:182-92.

6. Bertelsen L, Svendsen JH, Kober L, Haugan K, Hojberg S, Thomsen C, Vejlstrup N. Flow measurement at the aortic root - impact of location of through-plane phase contrast velocity mapping. J Cardiovasc Magn Reson 2016;18:55.

7. Wentland AL, Grist TM, Wieben O. Repeatability and internal consistency of abdominal 2D and 4D phase contrast MR flow measurements. Acad Radiol 2013;20:699-704.

8. Dyvorne H, Knight-Greenfield A, Jajamovich G, Besa C, Cui Y, Stalder A, Markl M, Taouli B. Abdominal 4D flow MR imaging in a breath hold: combination of spiral sampling and dynamic compressed sensing for highly accelerated acquisition. Radiology 2015;275:245-54.

9. da Silveira JS, Smyke M, Rich AV, Liu Y, Jin N, Scandling D, Dickerson JA, Rochitte CE, Raman SV, Potter LC, Ahmad R, Simonetti OP. Quantification of aortic stenosis diagnostic parameters: comparison of fast 3 direction and 1 direction phase contrast CMR and transthoracic echocardiography. J Cardiovasc Magn Reson 2017;19:35.

10. Dyverfeldt P, Bissell M, Barker AJ, Bolger AF, Carlhall
CJ, Ebbers T, Francios CJ, Frydrychowicz A, Geiger J, Giese D, Hope MD, Kilner PJ, Kozerke S, Myerson S, Neubauer S, Wieben O, Markl M. 4D flow cardiovascular magnetic resonance consensus statement. J Cardiovasc Magn Reson 2015;17:72.

11. Feneis JF, Kyubwa E, Atianzar K, Cheng JY, Alley MT, Vasanawala SS, Demaria AN, Hsiao A. 4D flow MRI quantification of mitral and tricuspid regurgitation: Reproducibility and consistency relative to conventional MRI. J Magn Reson Imaging 2018;48:1147-58.

12. Markl M, Wallis W, Harloff A. Reproducibility of flow and wall shear stress analysis using flow-sensitive fourdimensional MRI. J Magn Reson Imaging 2011;33:988-94.

13. Lantz J, Gupta V, Henriksson L, Karlsson M, Persson A, Carlhall CJ, Ebbers T. Intracardiac Flow at 4D CT: Comparison with 4D Flow MRI. Radiology 2018;289:51-8.

14. Hsiao A, Tariq U, Alley MT, Lustig M, Vasanawala SS. Inlet and outlet valve flow and regurgitant volume may be directly and reliably quantified with accelerated, volumetric phase-contrast MRI. J Magn Reson Imaging 2015;41:376-85.

15. Walheim J, Gotschy A, Kozerke S. On the limitations of partial Fourier acquisition in phase-contrast MRI of turbulent kinetic energy. Magn Reson Med 2019;81:514-23.

16. Komoriyama H, Tsuneta S, Oyama-Manabe N, Kamiya K, Nagai T. Four-dimensional flow magnetic resonance imaging visualizes significant changes in flow pattern and wall shear stress in the ascending aorta after transcatheter aortic valve implantation in a patient with severe aortic stenosis. Eur Heart J Cardiovasc Imaging 2020;21:21.

17. Reusz GS, Barczi A, Degi A, Cseprekal O, Kis E, Szabo A, Csoka M, Rudas G, Vegh A, Temmar M, Salvi P. Distance measurement for pulse wave velocity estimation in pediatric age: Comparison with intra-arterial path length. Atherosclerosis 2020;303:15-20.

18. Dyverfeldt P, Hope MD, Tseng EE, Saloner D. Magnetic resonance measurement of turbulent kinetic energy for the estimation of irreversible pressure loss in aortic stenosis. JACC Cardiovasc Imaging 2013;6:64-71.

19. Donati F, Figueroa CA, Smith NP, Lamata P, Nordsletten DA. Non-invasive pressure difference estimation from PC-MRI using the work-energy equation. Medical Image Analysis 2015;26:159-72.

20. Azarine A, Garcon P, Stansal A, Canepa N, Angelopoulos G, Silvera S, Sidi D, Marteau V, Zins M. Four-dimensional Flow MRI: Principles and Cardiovascular Applications. Radiographics 2019;39:632-48. 
21. Wentland AL, Wieben O, Francois CJ, Boncyk C, Munoz Del Rio A, Johnson KM, Grist TM, Frydrychowicz A. Aortic pulse wave velocity measurements with undersampled 4D flow-sensitive MRI: comparison with 2D and algorithm determination. J Magn Reson Imaging 2013;37:853-9.

22. Harloff A, Mirzaee H, Lodemann T, Hagenlocher P, Wehrum T, Stuplich J, Hennemuth A, Hennig J, Grundmann S, Vach W. Determination of aortic stiffness using 4D flow cardiovascular magnetic resonance a population-based study. J Cardiovasc Magn Reson 2018;20:43.

23. Verhagen JMA, Kempers M, Cozijnsen L, Bouma BJ, Duijnhouwer AL, Post JG, Hilhorst-Hofstee Y, Bekkers S, Kerstjens-Frederikse WS, van Brakel TJ, Lambermon E, Wessels MW, Loeys BL, Roos-Hesselink JW, van de Laar I, National Working Group on BAV, Taa. Expert consensus recommendations on the cardiogenetic care for patients with thoracic aortic disease and their first-degree relatives. Int J Cardiol 2018;258:243-8.

24. Farag ES, van Ooij P, Planken RN, Dukker KCP, de Heer F, Bouma BJ, Robbers-Visser D, Groenink M, Nederveen AJ, de Mol B, Kluin J, Boekholdt SM. Aortic valve stenosis and aortic diameters determine the extent of increased wall shear stress in bicuspid aortic valve disease. J Magn Reson Imaging 2018;48:522-30.

25. Bürk J, Blanke P, Stankovic Z, Barker A, Russe M, Geiger J, Frydrychowicz A, Langer M, Markl M. Evaluation of $3 \mathrm{D}$ blood flow patterns and wall shear stress in the normal and dilated thoracic aorta using flow-sensitive 4D CMR. J Cardiovasc Magn Reson 2012;14:84.

26. Wehrum T, Lodemann T, Hagenlocher P, Stuplich J, Ngo BTT, Grundmann S, Hennemuth A, Hennig J, Harloff A. Age-related changes of right atrial morphology and inflow pattern assessed using 4D flow cardiovascular magnetic resonance: results of a population-based study. J Cardiovasc Magn Reson 2018;20:38.

27. Frydrychowicz A, Markl M, Hirtler D, Harloff A, Schlensak C, Geiger J, Stiller B, Arnold R. Aortic hemodynamics in patients with and without repair of aortic coarctation: in vivo analysis by $4 \mathrm{D}$ flow-sensitive magnetic resonance imaging. Invest Radiol 2011;46:317-25.

28. Riesenkampff E, Fernandes JF, Meier S, Goubergrits L, Kropf S, Schubert S, Berger F, Hennemuth A, Kuehne T. Pressure fields by flow-sensitive, 4D, velocity-encoded CMR in patients with aortic coarctation. JACC Cardiovasc Imaging 2014;7:920-6.

29. Saitta S, Pirola S, Piatti F, Votta E, Lucherini F,
Pluchinotta F, Carminati M, Lombardi M, Geppert C, Cuomo F, Figueroa CA, Xu XY, Redaelli A. Evaluation of 4D flow MRI-based non-invasive pressure assessment in aortic coarctations. J Biomech 2019;94:13-21.

30. de Beaufort HW, Shah DJ, Patel AP, Jackson MS, Spinelli D, Yang EY, Ghosn MG, Autry K, Igo SR, Lumsden AB, Little SH, Trimarchi S, Bismuth J. Four-dimensional flow cardiovascular magnetic resonance in aortic dissection: Assessment in an ex vivo model and preliminary clinical experience. J Thorac Cardiovasc Surg 2019;157:467-76.e1.

31. Allen BD, Aouad PJ, Burris NS, Rahsepar AA, Jarvis KB, Francois CJ, Barker AJ, Malaisrie SC, Carr JC, Collins JD, Markl M. Detection and Hemodynamic Evaluation of Flap Fenestrations in Type B Aortic Dissection with 4D Flow MRI: Comparison with Conventional MRI and CTA. Radiol Cardiothorac Imaging 2019;1:e180009.

32. Geiger J, Hirtler D, Gottfried K, Rahman O, Bollache E, Barker AJ, Markl M, Stiller B. Longitudinal Evaluation of Aortic Hemodynamics in Marfan Syndrome: New Insights from a 4D Flow Cardiovascular Magnetic Resonance Multi-Year Follow-Up Study. J Cardiovasc Magn Reson 2017;19:33.

33. Ramos JG, Fyrdahl A, Wieslander B, Reiter G, Reiter U, Jin N, Maret E, Eriksson M, Caidahl K, Sorensson P, Sigfridsson A, Ugander M. Cardiovascular magnetic resonance $4 \mathrm{D}$ flow analysis has a higher diagnostic yield than Doppler echocardiography for detecting increased pulmonary artery pressure. BMC Med Imaging 2020;20:28.

34. Reiter G, Reiter U, Kovacs G, Olschewski H, Fuchsjager $M$. Blood flow vortices along the main pulmonary artery measured with MR imaging for diagnosis of pulmonary hypertension. Radiology 2015;275:71-9.

35. Kheyfets VO, Schafer M, Podgorski CA, Schroeder JD, Browning J, Hertzberg J, Buckner JK, Hunter KS, Shandas R, Fenster BE. 4D magnetic resonance flow imaging for estimating pulmonary vascular resistance in pulmonary hypertension. J Magn Reson Imaging 2016;44:914-22.

36. Fernandes JF, Hammel JM, Zhou J, Xiao Y, Chen M, Alves R, Lof J, Grieve SM, Schuster A, Kuehne T, Kutty S. Right ventricular energetics and power in pulmonary regurgitation vs. stenosis using four dimensional phase contrast magnetic resonance. Int J Cardiol 2018;263:165-70.

37. Markl M, Lee DC, Ng J, Carr M, Carr J, Goldberger JJ. Left Atrial 4-Dimensional Flow Magnetic Resonance Imaging: Stasis and Velocity Mapping in Patients With Atrial Fibrillation. Invest Radiol 2016;51:147-54. 
38. Cibis M, Lindahl TL, Ebbers T, Karlsson LO, Carlhall CJ. Left Atrial 4D Blood Flow Dynamics and Hemostasis following Electrical Cardioversion of Atrial Fibrillation. Front Physiol 2017;8:1052.

39. Föll D, Taeger S, Bode C, Jung B, Markl M. Age, gender, blood pressure, and ventricular geometry influence normal 3D blood flow characteristics in the left heart. Eur Heart J Cardiovasc Imaging 2013;14:366-73.

40. Svalbring E, Fredriksson A, Eriksson J, Dyverfeldt P, Ebbers T, Bolger AF, Engvall J, Carlhall CJ. Altered Diastolic Flow Patterns and Kinetic Energy in Subtle Left Ventricular Remodeling and Dysfunction Detected by 4D Flow MRI. PLoS One 2016;11:e0161391.

41. Kanski M, Arvidsson PM, Toger J, Borgquist R, Heiberg E, Carlsson M, Arheden H. Left ventricular fluid kinetic energy time curves in heart failure from cardiovascular magnetic resonance 4D flow data. J Cardiovasc Magn Reson 2015;17:111.

42. Schäfer M, Humphries S, Stenmark KR, Kheyfets VO, Buckner JK, Hunter KS, Fenster BE. 4D-flow cardiac magnetic resonance-derived vorticity is sensitive marker of left ventricular diastolic dysfunction in patients with mildto-moderate chronic obstructive pulmonary disease. Eur Heart J Cardiovasc Imaging 2018;19:415-24.

43. Barker N, Fidock B, Johns CS, Kaur H, Archer G, Rajaram S, Hill C, Thomas S, Karunasaagarar K, Capener D, AlMohammad A, Rothman A, Kiely DG, Swift AJ, Wild JM, Garg P. A Systematic Review of Right Ventricular Diastolic Assessment by 4D Flow CMR. BioMed Res Int 2019;2019:6074984.

44. Barker N, Zafar H, Fidock B, Elhawaz A, Al-Mohammad A, Rothman A, Kiely DG, van der Geest RJ, Westenberg J, Swift AJ, Wild JM, Plein S, Garg P. Age-associated changes in 4D flow CMR derived Tricuspid Valvular Flow and Right Ventricular Blood Flow Kinetic Energy. Sci Rep 2020;10:9908.

45. Crandon S, Westenberg JJM, Swoboda PP, Fent GJ, Foley JRJ, Chew PG, Brown LAE, Saunderson C, Al-Mohammad A, Greenwood JP, van der Geest RJ, Dall'Armellina E, Plein S, Garg P. Impact of Age and Diastolic Function on Novel, 4D flow CMR Biomarkers of Left Ventricular Blood Flow Kinetic Energy. Sci Rep 2018;8:14436.

46. Corrado PA, Macdonald JA, Francois CJ, Aggarwal NR, Weinsaft JW, Wieben O. Reduced regional flow in the left ventricle after anterior acute myocardial infarction: a case control study using 4D flow MRI. BMC Med Imaging 2019;19:101.
47. Garg P, Crandon S, Swoboda PP, Fent GJ, Foley JRJ, Chew PG, Brown LAE, Vijayan S, Hassell M, Nijveldt R, Bissell M, Elbaz MSM, Al-Mohammad A, Westenberg JJM, Greenwood JP, van der Geest RJ, Plein S, Dall'Armellina E. Left ventricular blood flow kinetic energy after myocardial infarction - insights from 4D flow cardiovascular magnetic resonance. J Cardiovasc Magn Reson 2018;20:61.

48. Garg P, van der Geest RJ, Swoboda PP, Crandon S, Fent GJ, Foley JRJ, Dobson LE, Al Musa T, Onciul S, Vijayan S, Chew PG, Brown LAE, Bissell M, Hassell M, Nijveldt R, Elbaz MSM, Westenberg JJM, Dall'Armellina E, Greenwood JP, Plein S. Left ventricular thrombus formation in myocardial infarction is associated with altered left ventricular blood flow energetics. Eur Heart J Cardiovasc Imaging 2019;20:108-17.

49. van Ooij P, Allen BD, Contaldi C, Garcia J, Collins J, Carr J, Choudhury L, Bonow RO, Barker AJ, Markl M. 4D flow MRI and T1-Mapping: Assessment of altered cardiac hemodynamics and extracellular volume fraction in hypertrophic cardiomyopathy. J Magn Reson Imaging 2016;43:107-14.

50. Martínez-Legazpi P, Bermejo J, Benito Y, Yotti R, Perez Del Villar C, Gonzalez-Mansilla A, Barrio A, Villacorta E, Sanchez PL, Fernandez-Aviles F, del Alamo JC. Contribution of the diastolic vortex ring to left ventricular filling. J Am Coll Cardiol 2014;64:1711-21.

51. Eriksson J, Bolger AF, Ebbers T, Carlhall CJ. Fourdimensional blood flow-specific markers of $\mathrm{LV}$ dysfunction in dilated cardiomyopathy. Eur Heart J Cardiovasc Imaging 2013;14:417-24.

52. François CJ, Srinivasan S, Schiebler ML, Reeder SB, Niespodzany E, Landgraf BR, Wieben O, Frydrychowicz A. 4D cardiovascular magnetic resonance velocity mapping of alterations of right heart flow patterns and main pulmonary artery hemodynamics in tetralogy of Fallot. J Cardiovasc Magn Reson 2012;14:16.

53. Sjöberg P, Bidhult S, Bock J, Heiberg E, Arheden H, Gustafsson R, Nozohoor S, Carlsson M. Disturbed left and right ventricular kinetic energy in patients with repaired tetralogy of Fallot: pathophysiological insights using 4D-flow MRI. Eur Radiol 2018;28:4066-76.

54. Hu L, Ouyang R, Sun A, Wang Q, Guo C, Peng Y, Qin Y, Zhang Y, Xiang Y, Zhong Y. Pulmonary artery hemodynamic assessment of blood flow characteristics in repaired tetralogy of Fallot patients versus healthy child volunteers. Quant Imaging Med Surg 2020;10:921-33. 55. Myerson SG. Heart valve disease: investigation by 
cardiovascular magnetic resonance. J Cardiovasc Magn

Reson 2012;14:7.

56. Chelu RG, Horowitz M, Sucha D, Kardys I, Ingremeau D, Vasanawala S, Nieman K, Paul JF, Hsiao A. Evaluation of atrial septal defects with 4D flow MRI-multilevel and inter-reader reproducibility for quantification of shunt severity. MAGMA 2019;32:269-79.

57. Valverde I, Nordmeyer S, Uribe S, Greil G, Berger F, Kuehne T, Beerbaum P. Systemic-to-pulmonary collateral flow in patients with palliated univentricular heart physiology: measurement using cardiovascular magnetic resonance 4D velocity acquisition. J Cardiovasc Magn Reson 2012;14:25.

58. Sjöberg P, Heiberg E, Wingren P, Ramgren Johansson J, Malm T, Arheden H, Liuba P, Carlsson M. Decreased Diastolic Ventricular Kinetic Energy in Young Patients with Fontan Circulation Demonstrated by FourDimensional Cardiac Magnetic Resonance Imaging. Pediatr Cardiol 2017;38:669-80.

59. Kamphuis VP, Elbaz MSM, van den Boogaard PJ, Kroft LJM, van der Geest RJ, de Roos A, Helbing WA, Blom NA, Westenberg JJM, Roest AAW. Disproportionate intraventricular viscous energy loss in Fontan patients: analysis by 4D flow MRI. Eur Heart J Cardiovasc Imaging 2019;20:323-33.

60. Schnell S, Entezari P, Mahadewia RJ, Malaisrie SC, McCarthy PM, Collins JD, Carr J, Markl M. Improved Semiautomated 4D Flow MRI Analysis in the Aorta in Patients With Congenital Aortic Valve Anomalies Versus Tricuspid Aortic Valves. J Comput Assist Tomogr 2016;40:102-8.

61. Alvarez A, Martinez V, Pizarro G, Recio M, Cabrera JA. Clinical use of 4D flow MRI for quantification of aortic regurgitation. Open Heart 2020;7:e001158.

62. Harris AW, Krieger EV, Kim M, Cawley PJ, Owens DS, Hamilton-Craig C, Maki J, Otto CM. Cardiac Magnetic Resonance Imaging Versus Transthoracic Echocardiography for Prediction of Outcomes in Chronic Aortic or Mitral Regurgitation. Am J Cardiol 2017;119:1074-81.

63. Rodríguez-Palomares JF, Dux-Santoy L, Guala A, Kale R, Maldonado G, Teixido-Tura G, Galian L, Huguet M, Valente F, Gutierrez L, Gonzalez-Alujas T, Johnson KM, Wieben O, Garcia-Dorado D, Evangelista A. Aortic flow patterns and wall shear stress maps by 4D-flow cardiovascular magnetic resonance in the assessment of aortic dilatation in bicuspid aortic valve disease. $\mathrm{J}$ Cardiovasc Magn Reson 2018;20:28.
64. Garg P, Swift AJ, Zhong L, Carlhall CJ, Ebbers T, Westenberg J, Hope MD, Bucciarelli-Ducci C, Bax JJ, Myerson SG. Assessment of mitral valve regurgitation by cardiovascular magnetic resonance imaging. Nat Rev Cardiol 2020;17:298-312.

65. Roes SD, Hammer S, van der Geest RJ, Marsan NA, Bax JJ, Lamb HJ, Reiber JH, de Roos A, Westenberg JJ. Flow assessment through four heart valves simultaneously using 3-dimensional 3-directional velocity-encoded magnetic resonance imaging with retrospective valve tracking in healthy volunteers and patients with valvular regurgitation. Invest Radiol 2009;44:669-75.

66. Chaturvedi A, Hamilton-Craig C, Cawley PJ, Mitsumori LM, Otto CM, Maki JH. Quantitating aortic regurgitation by cardiovascular magnetic resonance: significant variations due to slice location and breath holding. Eur Radiol 2016;26:3180-9.

67. Westenberg JJ, Roes SD, Ajmone Marsan N, Binnendijk NM, Doornbos J, Bax JJ, Reiber JH, de Roos A, van der Geest RJ. Mitral valve and tricuspid valve blood flow: accurate quantification with $3 \mathrm{D}$ velocity-encoded $\mathrm{MR}$ imaging with retrospective valve tracking. Radiology 2008;249:792-800.

68. Belhajer Z, Soulat G, Azarine A, Pontnau F, Ladouceur M, Bonnet D, Iserin L, Mousseaux E. Accuracy and Inter observer variability of blood flow quantification on 4D flow MRI in adult with transposition of the great arteries corrected by arterial switch. Journal of Cardiovascular Magnetic Resonance 2016;18(S1).

69. Crandon S, Elbaz MSM, Westenberg JJM, van der Geest RJ, Plein S, Garg P. Clinical applications of intra-cardiac four-dimensional flow cardiovascular magnetic resonance: A systematic review. Int J Cardiol 2017;249:486-93.

70. Johnson KM, Lum DP, Turski PA, Block WF, Mistretta CA, Wieben O. Improved 3D phase contrast MRI with off-resonance corrected dual echo VIPR. Magn Reson Med 2008;60:1329-36.

71. Jung B, Stalder AF, Bauer S, Markl M. On the undersampling strategies to accelerate time-resolved 3D imaging using k-t-GRAPPA. Magn Reson Med 2011;66:966-75.

72. Tsao J, Boesiger P, Pruessmann KP. k-t BLAST and k-t SENSE: dynamic MRI with high frame rate exploiting spatiotemporal correlations. Magn Reson Med 2003;50:1031-42.

73. Schnell S, Markl M, Entezari P, Mahadewia RJ, Semaan E, Stankovic Z, Collins J, Carr J, Jung B. k-t GRAPPA accelerated four-dimensional flow MRI in the aorta: effect 
on scan time, image quality, and quantification of flow and wall shear stress. Magn Reson Med 2014;72:522-33.

74. Stankovic Z, Fink J, Collins JD, Semaan E, Russe MF, Carr JC, Markl M, Langer M, Jung B. K-t GRAPPAaccelerated 4D flow MRI of liver hemodynamics: influence of different acceleration factors on qualitative and quantitative assessment of blood flow. MAGMA 2015;28:149-59.

75. Jung B, Honal M, Ullmann P, Hennig J, Markl M. Highly k-t-space-accelerated phase-contrast MRI. Magn Reson Med 2008;60:1169-77.

76. Ebel S, Dufke J, Kohler B, Preim B, Rosemeier S, Jung B, Dahnert I, Lurz P, Borger M, Grothoff M, Gutberlet M. Comparison of two accelerated 4D-flow sequences for aortic flow quantification. Sci Rep 2019;9:8643.

77. Bock J, Toger J, Bidhult S, Markenroth Bloch K, Arvidsson P, Kanski M, Arheden H, Testud F, Greiser A, Heiberg E, Carlsson M. Validation and reproducibility of cardiovascular 4D-flow MRI from two vendors using $2 \mathrm{x}$ 2 parallel imaging acceleration in pulsatile flow phantom and in vivo with and without respiratory gating. Acta Radiologica 2019;60:327-37.

78. Walheim J, Dillinger H, Gotschy A, Kozerke S. 5D Flow Tensor MRI to Efficiently Map Reynolds Stresses of Aortic Blood Flow In-Vivo. Sci Rep 2019;9:18794.

79. Walheim J, Dillinger H, Kozerke S. Multipoint 5D flow

Cite this article as: Zhuang B, Sirajuddin A, Zhao S, Lu M. The role of 4D flow MRI for clinical applications in cardiovascular disease: current status and future perspectives. Quant Imaging Med Surg 2021;11(9):4193-4210. doi: 10.21037/ qims-20-1234 cardiovascular magnetic resonance - accelerated cardiacand respiratory-motion resolved mapping of mean and turbulent velocities. J Cardiovasc Magn Reson 2019;21:42.

80. Retson TA, Besser AH, Sall S, Golden D, Hsiao A. Machine Learning and Deep Neural Networks in Thoracic and Cardiovascular Imaging. J Thorac Imaging 2019;34:192-201.

81. Avendi MR, Kheradvar A, Jafarkhani H. Automatic segmentation of the right ventricle from cardiac MRI using a learning-based approach. Magn Reson Med 2017;78:2439-48.

82. Winther HB, Hundt C, Schmidt B, Czerner C, Bauersachs J, Wacker F, Vogel-Claussen J. v-net: Deep Learning for Generalized Biventricular Mass and Function Parameters Using Multicenter Cardiac MRI Data. JACC Cardiovascular Imaging 2018;11:1036-8.

83. Tan LK, McLaughlin RA, Lim E, Abdul Aziz YF, Liew YM. Fully automated segmentation of the left ventricle in cine cardiac MRI using neural network regression. J Magn Reson Imaging 2018;48:140-52.

84. Berhane H, Scott M, Elbaz M, Jarvis K, McCarthy P, Carr J, Malaisrie C, Avery R, Barker AJ, Robinson JD, Rigsby CK, Markl M. Fully automated 3D aortic segmentation of 4D flow MRI for hemodynamic analysis using deep learning. Magn Reson Med 2020;84:2204-18. 